\title{
HELMINTOS INTESTINAIS EM CAVALOS DE TRABALHO E DE LAZER DE PORTO ALEGRE/RS
}

\author{
PICCOLI, Carmela ${ }^{1}$ \\ MARQUES, Sandra Márcia TieTz² \\ APPEL, Gabriela ${ }^{3}$; \\ SILVEIRA, Elissandra da ${ }^{4}$; \\ SIQUEIRA, Gabriela Bernardino ${ }^{3}$; \\ LOOS, Daiene Elisa ${ }^{5}$ \\ MATTOS, Mary Jane Tweedie de ${ }^{6}$.
} Veterinária/FAVET/UFRGS, ${ }^{3}$ Alunas de Graduação em Medicina Veterinária/FAVET/UFRGS, ${ }^{4}$ Médica Veterinária Residente Hospital de Clínicas Veterinárias/HCV/UFRGS, ${ }^{5}$ Médica Veterinária e Mestranda em Medicina de Equinos/HCV/FAVET/UFRGS, ${ }^{6}$ Professora Adjunta do Departamento de Patologia Clínica Veterinária/FAVET/UFRGS.

\section{RESUMO}

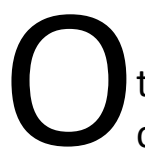
objetivo deste estudo foi avaliar a ocorrência de helmintos intestinais em cavalos de trabalho utilizados em carroças na coleta de resíduos recicláveis e comparar com cavalos de lazer criados no município de Porto Alegre, Rio Grande do Sul, através de exames parasitológicos executados nos anos de 2009 a 2012. Foram incluídos nesta investigação 276 cavalos, 131 animais de trabalho do Projeto Carroceiro e 145 cavalos utilizados para lazer. Amostras fecais foram coletadas e processadas pela técnica de flutuação com solução de cloreto de sódio ( $D=1.20)$. A positividade foi de $73 \%(202 / 276)$, com $64,8 \%(94 / 145)$ e $82,4 \%$ (108/131), respectivamente, para cavalos de lazer e de trabalho, com maior frequência de ovos da família Strongylidae e baixa ocorrência de Parascaris equorum, Strongyloides westeri e Anoplocephala spp. Nas duas categorias a positividade foi alta mostrando que medidas profiláticas, terapêuticas e orientação aos proprietários quanto ao controle da verminose equina são necessárias.

Palavras-chave: Equinos. Helmintos. Strongyloidea. Prevalência. 


\section{INTRODUÇÃO}

O cavalo está presente com o homem desde os primórdios da domesticação, nas funções de cavalaria montada, trabalho e lazer. Segundo a Confederação Nacional da Agricultura (CNA), o Brasil é dono da segunda maior tropa equina do mundo, com 5,9 milhões de cabeças. O agronegócio brasileiro do cavalo movimenta $\mathrm{R} \$$ 7,3 bilhões com geração de 640 mil empregos e o Brasil é um dos principais exportadores mundiais de carne de cavalo, com vendas externas que atingiram em 2008, US\$ 27,7 milhões (VIEIRA, 2012). O efetivo de equinos no Brasil aponta o Rio Grande do Sul em terceiro lugar (8,5\%), sendo que Minas Gerais e Bahia ocupam, respectivamente, o primeiro $(14,5 \%)$ e o segundo lugar $(10,6 \%)$ (IBGE, 2010).

Entre as patologias que afetam a sanidade de cavalos, as infecções parasitárias são relatadas em equinos ao redor do mundo, sendo importante causa de morbidade e mortalidade (ANDRADE et al., 2009; BOTELHO et al., 2012; KORNÁS et al., 2010; MORALES et al., 2011). Cavalos são parasitados por mais de 90 espécies de helmintos e duas subfamílias de nematóides intestinais, os grandes estrôngilos (Strongylinae) e os pequenos estrôngilos (Cyatostominae) são de grande relevância na clínica equina, em termos de prevalência e patogenicidade. Parascaris equorum apresenta distribuição mundial e possui importância patogênica dependendo do manejo, faixa etária e controle, enquanto Strongyloides westeri tem baixa significância clínica, e em animais mais velhos a infecção é inaparente, entretanto sintomática em animais jovens (ROBERTS; JANOVY JUNIOR, 2009).

O Município de Porto Alegre criou o Programa de Redução Gradativa do Número de Veículos de Tração Animal (Lei no 10.531, de 10 de setembro de 2008), com o cadastramento de todos os condutores de veículos de tração animal e a extinção total destes veículos nas ruas da cidade até 2016. Os animais do projeto carroceiro são aqueles utilizados para trabalho de tração em veículos denominados carroças, na coleta de resíduos recicláveis de todos os bairros da cidade de Porto Alegre. Aqueles animais que precisam de um atendimento hospitalar são encaminhados para o Hospital Veterinário da Universidade Federal do Rio Grande do Sul (UFRGS), cadastrados no Projeto Carroceiro, recebendo atendimento gratuito 
nas diferentes especialidades. O Laboratório de Helmintoses participa deste projeto desde 2009, como ação de extensão universitária, executando o diagnóstico parasitológico.

O objetivo deste trabalho foi avaliar a prevalência de helmintos intestinais em cavalos de trabalho e de lazer criados no município de Porto Alegre, estado do Rio Grande do Sul, através de exames coprológicos executados nos anos de 2009 até 2012.

\section{MATERIAL E MÉTODOS}

Foram incluídos nesta investigação 276 equinos adultos, dos quais 131 cavalos do Projeto Carroceiro e 145 cavalos utilizados para lazer de estabelecimentos particulares. As amostras fecais dos cavalos utilizados para lazer foram colhidas em visitas aos sítios da cidade de Porto Alegre e dos cavalos do projeto colhidas no Hospital Veterinário da UFRGS, no período de quatro anos (2009-2012).

As amostras fecais foram coletadas diretamente da ampola retal, acondicionadas em frascos plásticos com tampa e encaminhadas ao Laboratório de Helmintoses da FAVET/UFRGS. Para a verificação da infecção parasitária, as fezes foram processadas pela técnica qualitativa de Willis-Molay (HOFFMAN, 1987). A leitura das lâminas foi executada com microscópio óptico Nikon, em aumento de 10X e 20X. Os resultados foram anotados em fichas próprias e encaminhados para o corpo clínico do Hospital Veterinário que executou o tratamento antihelmíntico e orientou a cerca das práticas de manejo sanitário. No caso dos cavalos de lazer, os proprietários retiraram os resultados no laboratório.

\section{RESULTADOS E DISCUSSÃO}

A prevalência de exames parasitológicos positivos foi de 73\% (202/276), com 64,8\% (94/145) e $82,4 \%$ (108/131), respectivamente, para cavalos de lazer e de trabalho. Os percentuais de positividade foram semelhantes para machos e fêmeas. A Figura 1 mostra os resultados para os dois grupos de cavalos, no período investigado. A dominância da infecção ocorreu por ovos da família Strongylidae (96,03\%) enquanto poli-infecção com Parascaris equorum foi de $4,2 \%$ e $7,4 \%$, respectivamente, para cavalos de lazer e de trabalho. A baixa ocorrência de $S$. 
westeri com Strongylidae foi maior $(8,5 \%)$ em cavalos de lazer do que em animais de trabalho (5,5\%) bem como para ovos de Anoplocephala spp., 6,4\% e 3,7\%, respectivamente, para as mesmas categorias de animais.

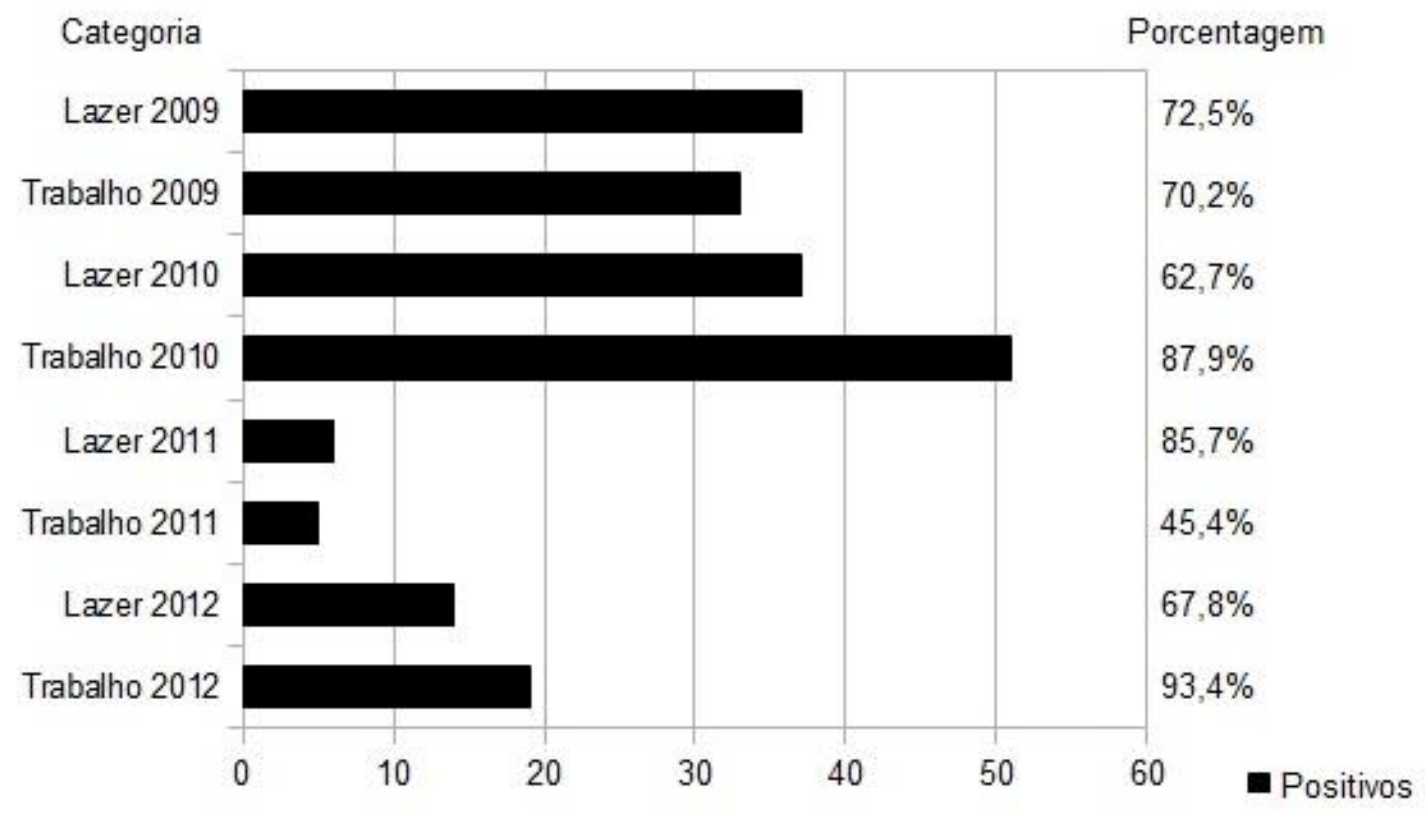

Figura 1 - Número e porcentagem de animais positivos em exames parasitológicos de cavalos de lazer e trabalho de Porto Alegre/RS, no período de janeiro de 2009 a dezembro de 2012.

Nesta investigação detectou-se uma alta prevalência (73\%) de cavalos portadores de ovos de helmintos intestinais. Notadamente a infecção mais prevalente ocorreu por ovos da família Strongylidae (96,03\%). Entretanto, na avaliação do período de quatro anos, poucos animais mostraram poli-infecção com Parascaris equorum, com S. westeri e com Anoplocephala spp., semelhante aos resultados de Ferraro et al. (2008), Andrade et al. (2009) e Morales et al. (2011).

Na comparação com os equinos oriundos de apreensão em logradouros públicos e rodovias federais no Rio de Janeiro, Martins et al. (2002) evidenciaram prevalência maior para $P$. equorum (20\%) e S. westeri $(6,7 \%)$ e resultado semelhante para Strongylus spp. e igual proporção para machos e fêmeas; todavia em cavalos adultos e criados para lazer em Seropédica (RJ) e áreas vizinhas, a prevalência foi de $48 \%$, com animais desverminados 
regularmente e clinicamente sadios (Botelho et al., 2012). No Paraná, um levantamento baseado em amostras fecais de 41 cavalos mostrou infecção por estrongilídeos em $88 \%$ dos animais e pouca frequência associada a $P$. equorum (FERRARO et al., 2008). Belmonte et al. (2009) analisaram amostras fecais de 40 cavalos de área rural de Caçapava do Sul (RS) em 2007, registrando infecção moderada (OPG médio 435, grupo A com 10 animais) e alta (OPG médio 708, grupo C com 10 animais) por parasitos da família Strongylidae. No nordeste brasileiro, na cidade de Aracaju (SE), Andrade et al. (2009) avaliaram infecção parasitária em cavalos de tração atendidos na Clínica Médica do Hospital Veterinário e constataram $58 \%$ de animais parasitados e Strongylidae foi a mais prevalente, infectando todos os animais positivos, enquanto Oxyuris equi $(17,24 \%)$ e $P$. equorum $(3,45 \%)$ tiveram percentuais baixos, semelhantes ao detectado nesta investigação. Entretanto, Ahid et al. (2009) investigando infecção helmíntica em cavalos do semiárido nordestino, em Mossoró (RN), detectaram ovos de $P$. equorum (20\%) em uma população maior de cavalos do que o registrado em Porto Alegre. Lagaggio et al. (2012) detectaram que 60\% dos cavalos estabulados na hípica da Universidade Federal de Santa Maria (RS) apresentaram ovos da superfamília Strongyloidea no exame das fezes e $10 \%$ estavam infectados com ovos da superfamília Ascaroidea. Em Curitiba (PR), Hein et al. (2012) avaliaram amostras fecais de 30 equinos de uma vila na periferia da cidade e detectaram $90 \%$ de positividade com predomínio de $23,3 \%$ de ovos da família Strongylidae.

Nos diversos estudos constata-se a família Strongylidae com predomínio, mesmo nas avaliações de animais que haviam recebido algum tratamento anti-helmíntico, denotando falha nas estratégias anti-helmínticas e sanitárias, afirmativa constatada por Morales et al. (2011) em avaliação da carga parasitária em animais com plano de desverminações, cuja prevalência foi de $73 \%$ de ovos de Strongylus spp. e de $4 \%$ para $P$. equorum, concluindo que a carga parasitária alta sugere plano sanitário inadequado e de baixa especificidade no controle parasitário, além de uma possível resistência associada ao clima que altera o ciclo parasitário.

Neste estudo, animais de trabalho e sem nenhum tratamento anti-helmíntico superaram em positividade cavalos de lazer, cujos proprietários adotaram rotina de desverminação bianual 
e, neste grupo a positividade foi acima de $50 \%$, podendo caracterizar resposta não eficaz ao vermífugo ou rotina anti-helmíntica incorreta, concordando com Morales et al. (2011). Além disso, em animais sob trabalho intenso e nem sempre com rotina alimentar adequada, devido principalmente a longas distâncias percorridas, poderiam sugerir maior efeito negativo decorrente de infecção parasitária. Entretanto, os efeitos do exercício e do treinamento e a rotina alimentar sobre as funções gastrintestinais e suas alterações ainda não são completamente conhecidas, apenas descrito por White (1995), relacionando o excesso de exercícios físicos ou a sua falta poderem estar vinculados com a síndrome cólica, causada por S. vulgaris e por formar trombos nas artérias mesentéricas durante a migração larval.

Contrapondo a afirmação de resistência anti-helmíntica, Gawor (1995) avaliou, através de necropsia, 50 intestinos de cavalos, constatando P. equorum (26\%) e S. westeri (4\%), porém credita a ausência de estrongilídeos devido às medicações anti-helmínticas, enquanto Kornás et al. (2010), em relato de 11 anos de exames parasitológicos registraram $P$. equorum, Anoplocephala spp. e pequenos estrôngilos, sugerindo que em animais com acesso ao pasto o controle de nematódeos é mais difícil. Além destas variáveis, a cama das baias e os cochos de alimentação, se não são submetidos a manejo higiênico adequado, permitem a permanência de formas parasitárias, o que foi avaliado por Lagaggio et al. (2012), quando detectaram formas parasitárias em 100\% das camas, com maior prevalência de ovos de helmintos (78\%) e dentre estes, ovos do tipo Strongyloidea (56\%), Ascaroidea (32\%) e Rhabdiasoidea (12\%), 7\% de larvas de helmintos, 6\% de cistos de Giardia spp., 4\% de ovos de Taenia spp. e $2 \%$ de oocistos de protozoários.

\section{CONCLUSÃO}

Este estudo de quatro anos confirma o caráter cosmopolita dos principais endoparasitos de equinos. Ovos de helmintos da família Strongylidae foram dominantes nos dois grupos de cavalos. São necessárias avaliações parasitológicas rotineiras, plano de desverminações baseados no perfil da propriedade e orientação sobre o manejo higiênico dos animais. 


\title{
INTESTINAL HELMINTHS IN WORK AND LEISURE HORSES IN PORTO ALEGRE/RS
}

\begin{abstract}
The aim of this study was to evaluate the occurrence of intestinal helminths in working horses used in wains to collect recyclable waste and compare with leisure horses bred in the city of Porto Alegre, Rio Grande do Sul, through parasitologic tests executed between the years 2009 and 2012. 276 horses were included in this research, 131 animals from the project Carroceiro and 145 horses used for recreation. Faecal samples were collected and sent to the laboratory of helminthiasis of FAVET/UFRGS and were processed by the flotation technique with sodium chloride $(D=1.20)$. The positivity was $73 \%(202 / 276)$, with $64.8 \%$ (94/145) and $82.4 \%$ (108/131), respectively, for leisure and working horses, with a higher frequency of eggs of the family Strongylidae and low occurrence of Parascaris equorum, Strongyloides westeri and Anoplocephala spp. In both categories the positivity was high showing that therapeutic and prophylactic measures, besides guidance to owners regarding equine worm control are necessary.
\end{abstract}

Keywords: Equines. Helminths. Strongyloidea. Prevalence.

\section{HELMINTOS INTESTINALES EN CABALLOS DE TRABAJO Y DE RECREACIÓN DE PORTO ALEGRE/RS}

\section{RESUMEN}

$\mathrm{E}$ I objetivo de este estudio fue evaluar la ocurrencia de las helmintiasis intestinales en caballos de trabajo, utilizados en carros en la recolección de desechos reciclables y comparar con caballos de recreación criados en la ciudad de Porto Alegre, Rio Grande do Sul, a través de examen parasitológico ejecutado en los años 2009 y 2012. Se incluyeron en esta investigación 276 caballos, 131 animales del "Proyecto Carroceiro" y 145 caballos utilizados para la recreación. Se colectaron muestras fecales que fueron procesadas mediante la técnica de flotación con la solución de cloruro de sodio $(D=1.20)$. La positividad fue del $73 \%(202 / 276)$, con $64,8 \%$ (94/145) y el $82,4 \%(108 / 131)$, respectivamente, para caballos de recreación y de trabajo. Hubo mayor frecuencia de huevos de la familia Strongylidae y baja ocurrencia de Parascaris equorum, Strongyloides westeri y Anoplocephala spp. En ambas categorías la positividad fue alta y muestra que las medidas profilácticas, terapéuticas y orientación a los propietarios en cuanto al control de la parasitosis equina son necesarias.

Palabras clave: Equinos. Helmintos. Strongyloidea. Prevalencia. 


\section{REFERÊNCIAS}

ANDRADE, R. L. F. S.; SOBRAL, J. C.; SILVA, K. M. G. Avaliação clínica, hematológica e parasitária em equinos de tração na cidade de Aracajú, Sergipe. Acta Veterinária Brasílica, v. 3, n. 3, p. 138-142, 2009.

AHID, S. M. M.; SUASSUNA, A. C. D.; FILGUEIRA, K. D. Fauna parasitológica em animais domésticos e exóticos no município de Mossoró-RN. Biociências, Porto Alegre, v. 17, n. 1, p. 44-47, 2009.

BELMONTE, C.; FERNANDES, F.; GASPARY, J.; et al. Antiparasitários no controle de helmintos em cavalos mantidos em campo nativo na região central do Rio Grande do Sul, Brasil.

Veterinária em Foco, v. 7, n. 1, p. 46-51, 2009.

BOTELHO, G. G.; CASSIANO, A. L. V.; BOTELHO, C. F. M.; et al. Análise hematológica, bioquímico-sérica e coproparasitológica de equinos criados em Seropédica, RJ. Revista Brasileira de Medicina Veterinária, v. 34, n. 1, p. 69-72, 2012.

FERRARO, C. C.; KLOSS, A. B.; SOUZA, D. F.; et al. Prevalência parasitológica de cavalos de carroceiros em Curitiba, Paraná. Revista Brasileira de Parasitologia Veterinária, v. 17, supl. 1, p. 175-177, 2008.

GAWOR, J. J. The prevalence and abundance of internal parasites in working horses autopsied in Poland. Veterinary Parasitology, v. 58, p. 99-108, 1995.

HEIN, K. K.; YOKOYOMA, M. R.; SCHAULE, M. T.; et al. Verificação da ocorrência parasitológica com potencial zoonótico em fezes de equinos na Vila Osternack-Curitiba-PR. Revista Eletrônica da Faculdade Evangélica do Paraná, Curitiba, v. 2, n. 2, p. 71-79, 2012. Disponível em: http://www.fepar.edu.br/revistaeletronica/index.php/revfepar/article/view/49/61. Acesso em: 21 jan. 2014.

HOFFMAN, R. P. Diagnóstico de Parasitismo Veterinário. Porto Alegre: Sulina, 1987.

IBGE. Instituto Brasileiro de Geografia e Estatística. Produção da Pecuária Municipal. v. 38. 2010. Disponível em:

http://www.ibge.gov.br/home/estatistica/economia/ppm/2010/tabelas_pdf/tab12.pdf. Acesso em: 12 mar. 2014.

KORNÁS, S.; CABARET, J.; SKASKA, M.; et al. Horse infection with intestinal helminthes in relation to age, sex, access to grass and farm system. Veterinary Parasitology, v. 174, p. 285291, 2010. 
LAGAGGIO, V. R. A.; JORGE, L. L.; OLIVEIRA, V.; et al. Achados de formas parasitárias em camas de equinos-Santa Maria-RS/Brasil. Redevet, 2012. Disponível em:

http://www.redevet.com.br/artigos/camas.htm. Acesso em: 15 ago. 2013.

MARTINS, I. F.; CORREIA, T. R.; SOUZA, C. P.; et al. Frequência de nematóides intestinais de equinos oriundos de apreensão no Estado do Rio de Janeiro. Revista Brasileira de Parasitologia Veterinária, v. 10, n. 1, p. 37-40, 2002.

MORALES, B. A. A.; BELCO, H.; GÓMEZ, R. M. S. Prevalência de parásitos gastrintestinales en caballos pura sangue de carrera (Equus caballus) durante el periodo de cuarentena $2010 \mathrm{em}$ el Hipodromo "La Rinconada" Caracas, Venezuela. Neotropical Helminthology, v. 5, n. 1, p. 85-88, 2011.

ROBERTS, L. S.; JANOVY JUNIOR, J. Basic Principles and Concepts II: Immunology and Pathology. In: SCHMIDT, G. D.; ROBERTS, L. Fundations of Parasitology. McGraw-Hill Science/Engineering/Math, 2009. Cap. 3, p. 25-42.

VIEIRA, E. R. Aspectos econômicos e sociais do complexo do agronegócio cavalo no Estado de Minas Gerais. Belo Horizonte: UFMG, 2012. 140p. Dissertação de Mestrado, Escola de Veterinária, Universidade Federal de Minas Gerais, 2012. Disponível em:

http://www.conselhos.mg.gov.br/uploads/20/Aspectos\%20economicos\%20e\%20sociais\%20 -\%20cavalo\%20em\%20MG\%20(UFMG).pdf. Acesso em: 30 dez. 2013.

WHITE, N. A. Epidemiology of equine colic. In: CICLO INTERNACIONAL DE CÓLICA EQUINA, 2, UNESP-Jaboticabal, Anais. p. 1-9, 1995. 